\title{
Power Hotspots in Space: Powering CubeSats via Inter-Satellite Optical Wireless Power Transfer
}

\author{
Marwa Qaraqe, Member, IEEE, Muhammad Usman, Ahmet Serbes, Member, IEEE, \\ Imran Shafique Ansari, Member, IEEE, and Mohamed-Slim Alouini, Fellow, IEEE
}

\begin{abstract}
The desire to provide global Internet coverage is driving traditional terrestrial networks to evolve towards an integrated space and terrestrial network wherein miniaturized satellites, a.k.a. CubeSats, remain at the core. Although both academia and industry are investigating the potential of CubeSats to provide global connectivity, they still fall short of the power generation capacity to enable high data rate communication in gigabits per second range. This article spotlights on alternate sources of energy that can provide the power demands needed for high data rate communication. We present the concept of power HotSpots wherein the bigger satellites in low earth orbits (LEO), having power generation capacity much larger than CubeSats, can transfer their excess energy to CubeSats in need, using optical wireless technology. This provides a business opportunity for larger enterprises having the capability of launching bigger satellites to sell their power to CubeSats. As a proof of concept, this article presents a basic simulation regarding optical wireless power transfer (OWPT) to CubeSats. In addition, we highlight future research challenges in this area to maximize OWPT.
\end{abstract}

Index Terms-Free space optics, Optical wireless power transfer, Integrated space and terrestrial networks, CubeSats, Solar panels, Power storage.

\section{INTRODUCTION}

As the world is becoming more digital, the demand to connect the unconnected 3.9 billion people and increased demand of higher data-rates from the connected users [1] is becoming more critical. Although fifth generation (5G) mobile communication networks provide promising use-cases, such as massive connection and Internet-of-things (IoT) at low latency and higher data rates, it still has a coverage limitation similar to all traditional terrestrial networks. In parallel to terrestrial networks, complementary satellite networks exist that provide coverage to remote rural areas, vehicles traveling in the deep sea, and in-air communication. However, current satellite networks fall short of supporting many valuable usecases, which on the other hand, are inherently supported by contemporary terrestrial networks.

Marwa Qaraqe and Muhammad Usman are with the Division of Information and Computing Technology, College of Science and Engineering, Hamad Bin Khalifa University (HBKU), P.O. Box: 34110, Education City, Doha, Qatar (\{mqaraqe, musman\}@hbku.edu.qa).

Ahmet Serbes is currently with the Department of Electronics and Communications Engineering, Yildiz Technical University, Davutpasa, Istanbul, 34220, Turkiye. (e-mail: aserbes@yildiz.edu.tr).

Imran Shafique Ansari is currently with the School of Engineering, University of Glasgow, Glasgow G12 8QQ, United Kingdom (e-mail: imran.ansari@glasgow.ac.uk).

Mohamed-Slim Alouini is currently with the Computer Electrical, and Mathematical Science and Engineering (CEMSE) Division, King Abdullah University of Science and Technology (KAUST), Thuwal 23955-6900, Saudi Arabia, (e-mail: slim.alouini@kaust.edu.sa).

\section{A. Background}

Combining the positives of both terrestrial and satellite networks, an integrated space and terrestrial network (ISTN) is proposed to connect anything in the globe anywhere and at anytime with a desired quality of service (QoS) requirement [2]. An overview of an ISTN is presented in Fig. 1]

The ISTN can be utilized to provide high data rate coverage to the areas where conventional cellular networks are not available, such as antarctic glaciers, deep seas, remote forests (Amazon), deep deserts, and disaster scenarios where terrestrial networks have been completely or partially damaged.

This new global coverage will include applications from various industries, including but not limited to, communication, agriculture, manufacturing, energy, mining, logistics, environmental monitoring, eHealth, public safety, and defense, covering a full span of low latency and high throughput applications to high latency and low throughput applications. With the aim of providing seamless connectivity anywhere and anytime across the globe, the integration of space and terrestrial networks may become a pivotal aim within $6 \mathrm{G}$ [1]. On the other hand, given the cost and complexity of launching a satellite in space, it may become a challenge for both academia and industry to build real-time test-beds for such integrated $6 \mathrm{G}$ networks. However, the introduction of nano and pico satellites, such as CubeSats, has opened up new opportunities for researchers and small enterprises in recent decades. CubeSats are smaller satellites with a modular structure of one unit (1U) wherein $1 \mathrm{U}$ corresponds to a cube of $10 \mathrm{~cm} \times 10 \mathrm{~cm} \times 10 \mathrm{~cm}$. CubeSats are generally utilized for different missions in the low earth orbit (LEO), including environmental monitoring and communications.

\section{B. Motivation}

More recently, due to their low deployment cost and cheap connectivity, CubeSats are being considered as a potential solution to provide global Internet coverage [3]. For instance, KEPLER launched their KIPP CubeSats in 2018 to provide coverage at the north and south poles, offering $40 \mathrm{Mbits} / \mathrm{s}$ data rate in the Ku-band [3]. It is important to note that CubeSats do not aim to replace the bigger LEO constellations but complement them towards the goal of global connectivity. Given the popularity of CubeSats as a potential enabler of providing global connectivity, some works in the literature propose CubeSat-based cloud radio access for future cellular networks [4]. Such works provide grounds and inspirations 


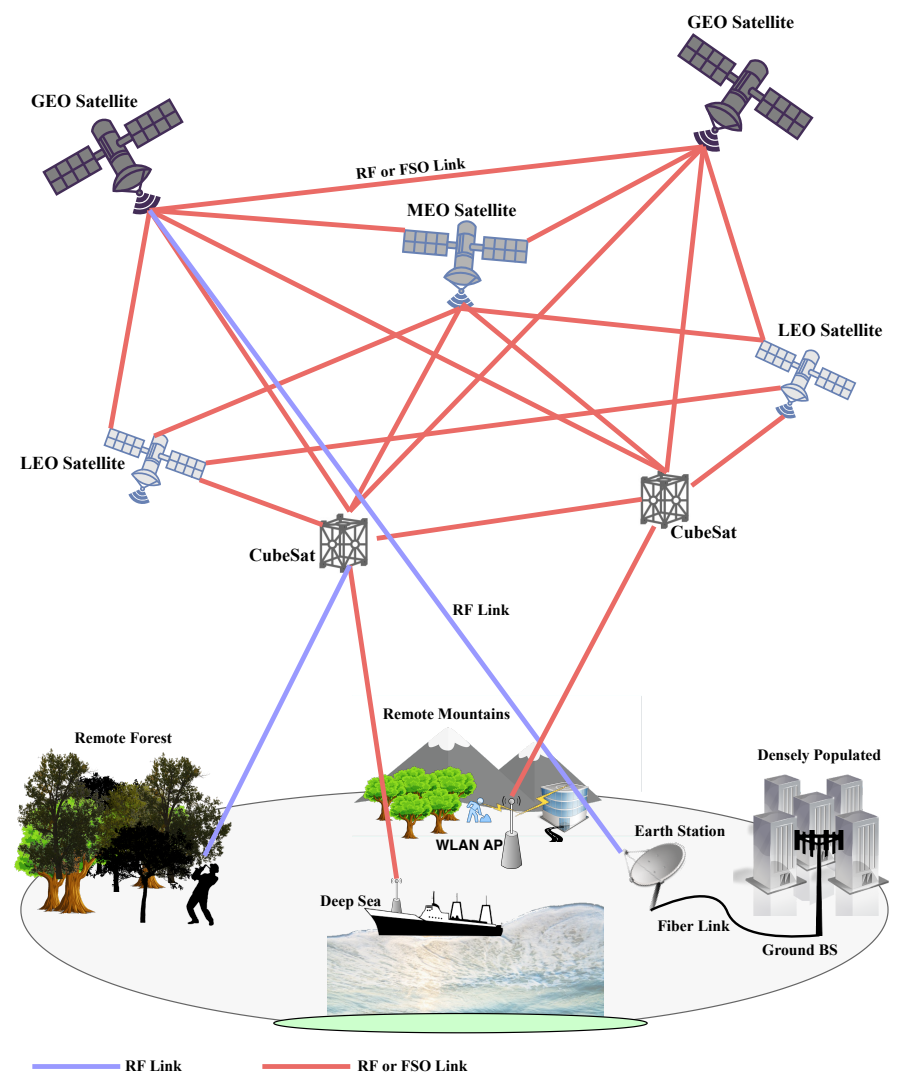

Fig. 1: A vision of ISTN in future $6 \mathrm{G}$ networks.

to research the design of high data rate connections between ground stations and CubeSats.

The power in CubeSats is mainly harvested via utilizing solar panels mounted on the sides of the CubeSat. However, given the size of a CubeSat, the on-board solar panels are potentially not enough to generate sufficient amount of energy for various power hungry applications including high datarate communication. In fact, the power generation capacity of a CubeSat may become a critical bottleneck to achieve the aim of providing global coverage at higher data rates. A potential solution to this power bottleneck problem would be to harvest energy from bigger LEO satellites that have a far greater capacity of generating and storing energy. Therefore, transferring power from larger LEOs to CubeSats may become a viable solution in numerous scenarios. These scenarios may include the need of communicating with a specific target on earth for a specific duration of time at high data-rate, or fast charging of a CubeSat's batteries.

Apart from higher data-rate communication requirements of CubeSats, another motivation of this in-space power transfer is the need of miniaturized thrusters in CubeSats to increase their service life. Orbiting in the lower orbits, CubeSats suffer from higher atmospherics drag, which results in deorbiting. This atmospheric drag requires miniaturized thrusters, which adds further burden on the already limited power supply in CubeSats. The power supplied from larger LEO satellites will not only enable high data rate communication but will also extend the life-time of CubeSat by supplying sufficient energy for orbit rising.

In this article, we analyze optical wireless power transfer (OWPT) 1 options in space between larger LEO satellites and CubeSats. We first identify potential challenges and key technologies for OWPT. Subsequently, we propose the concept of power HotSpots in space between larger LEOs and CubeSats. The power HotSpot is the physical range of a bigger LEO, where it can perform OWPT to a CubeSat. This concept may also provide business opportunities to enterprises capable of launching power-HotSpot-enabled satellites for commercial purposes providing extra energy to CubeSats orbiting Earth. This article also provides a preliminary OWPT proof of concept by presenting basic simulations. In addition, we elaborate on the research challenges for OWPT in space and discuss future research directions.

\section{Power Related Challenges at a CubeSat}

The vision provided in Fig. 1 enables CubeSat to establish links not only with the ground stations but also with the other satellites in the space. All of these communication links require power to communicate at higher data rates. To further understand the power-related challenges in CubeSats, this section highlights the challenges faced by CubeSats in terms of their power requirements, generation, and storage.

\footnotetext{
${ }^{1}$ An important concept in this context is simultaneous wireless information and power transfer (SWIPT) [5]. However, the focus of this work is power transfer only, considering that communication in an ISTN is already established.
} 


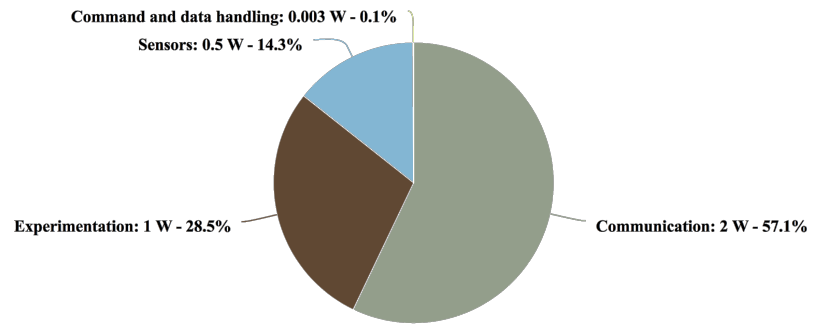

Fig. 2: Power requirements of a typical CubeSat subsystem communicating at a low data rate $(2 \mathrm{Mbps}$ at $500 \mathrm{MHz}$ frequency).

\section{A. Power Generation Capacity of a CubeSat}

Given the size of a CubeSat, generating a large amount of energy to enable Gbps communication between the CubeSat and the ground station becomes a real challenge.

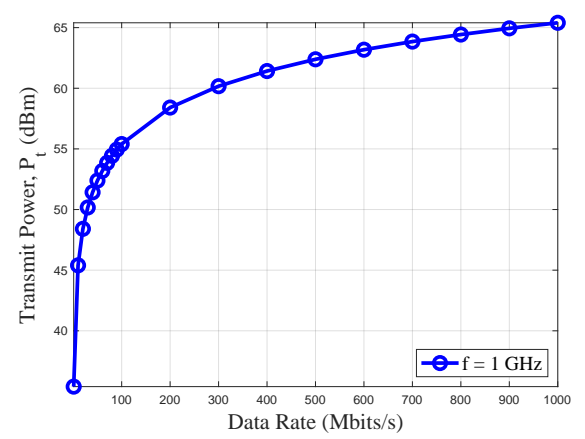

Fig. 3: Transmit power $P_{t}$ for a downlink at a CubeSat against different data rate requirements between a CubeSat and the ground station at a carrier frequency of $1 \mathrm{GHz}$.

CubeSats generally utilize solar panels to convert light into electricity to perform their day-to-day operations. However, due to the compact size of a CubeSat, the power generated from solar panels will generally not be sufficient for power hungry tasks. More recently, there have been some attempts to deploy high power solar panels over CubeSats. However, they still fall short of fulfilling the power requirements of high data-rate communication. For instance, HaWK (high Watts per kilogram) is a solar array recently designed for high power generation at CubeSat [6], whose two variants eHaWK and zHaWK provide peak powers of $84 \mathrm{~W}$ and $36 \mathrm{~W}$, respectively. However, this peak power is not sufficient to establish high data rate links between CubeSats and ground station at any given carrier frequency.

Traditionally, CubeSats use batteries for power when they are in an eclipse. A CubeSat orbiting at a distance of $600 \mathrm{Km}$ from earth generally completes one cycle in 90 minutes, with approximately 35 minutes of the 90 minutes in an eclipse [7], during which the batteries are used. This frequent charging and discharging of the batteries limits their lifetime.

\section{B. Power Requirements of a CubeSat}

The power distribution of a general CubeSat (mainly meant for experimentation and a very low data rate communication)
TABLE I: Physical Parameters

\begin{tabular}{|l|l|}
\hline Parameter Name & Physical Value \\
\hline \hline Carrier Frequency & $1 \mathrm{GHz}$ \\
\hline Transmit antenna gain $G_{t}$ & $15.5 \mathrm{dBi}$ \\
\hline Propagation path loss $L_{p}$ & $153.76 \mathrm{~dB}$ \\
\hline Receive antenna gain $G_{r}$ & $0 \mathrm{~dB}$ \\
\hline System noise temperature $\left(10 \log _{10} T_{s}\right)$ & $31.39 \mathrm{dBK}$ \\
\hline Boltzmann constant $\left(10 \log _{10} \mathrm{k}\right)$ & $-228 \mathrm{dBm} / \mathrm{K} / \mathrm{Hz}$ \\
\hline $\begin{array}{l}\text { SNR required for } 10^{-5} \mathrm{BER}(\mathrm{BPSK}, \mathrm{QPSK}, \\
\text { 8-FSK with FEC) }\end{array}$ & $>9.5 \mathrm{~dB}$ \\
\hline
\end{tabular}

among its subsystems is presented in Fig. 2. A $2 \mathrm{~W}$ of communication power is needed to communicate over a distance of $700 \mathrm{Km}$ at a frequency of $500 \mathrm{MHz}$ for a data rate of around $2 \mathrm{Mbits} / \mathrm{sec}$. However, the desire of higher data rates around Gbps requires larger power to be allocated to the communication infrastructure of the CubeSat. An estimation of the transmit power requirements at a CubeSat downlink is provided in Fig. 3, which employs (1) in [7] with the parameters provided in Table I for a bit error rate (BER) of $10^{-5}$. Here, (1) represents the free space path loss excluding any channel conditions. Hence, the required transmit powers presented in Fig. 3 represent the minimum required power. The signal-to-noise ratio (SNR) at the receiver, in $\mathrm{dB}$, is

$$
\begin{aligned}
S N R_{d B}= & 10 \log _{10} \frac{E_{b}}{N_{0}} \\
= & 10 \log _{10}\left(\frac{P_{t} G_{t} G_{r}}{k T_{s} R L_{p}}\right) \\
= & P_{t, d B m}-30+G_{t, d B i}-L_{P, d B} \\
& -10 \log _{10} k-10 \log _{10} T_{s}-10 \log _{10} R,
\end{aligned}
$$

where $R$ is the data rate of communication link and $P_{t, d B m}$ is required transmitted power at CubeSat in $\mathrm{dBm}$. Rest all the symbols in (1) are defined in Table [1 [7]. The communication at a CubeSat may be divided in three different scenarios, illustrated in Fig. 1. namely, (i) its communication to ground station, (ii) inter-CubeSat communication in LEO orbits, and (iii) inter-satellite communication among LEO, medium earth orbit (MEO), and geostationary equatorial orbit (GEO).The required transmit powers presented in Fig. 3 is for the first scenario, communication between the CubeSat and the ground station.

\section{Power Storage Limitations of a CubeSat}

As mentioned earlier, the storage of generated electrical power in a CubeSat is a real challenge. Lithium-ion ( $\mathrm{Li}$ ion) technology has become the favored energy storage option since the success of the twin Mars exploration rovers that were launched in 2003: Spirit and Opportunity. Li-ion is the common name for many types of different battery chemistry's, which include lithium compounds on the anode. Table II gives a comparison of the most widely used battery chemistry's. Superior specific energy and energy density has led Li-ion batteries to become the first choice for the energy storage of the most of NASA's applications.

It should be noted that most of the Li-ion batteries provide lower performance when affected by the harsh space conditions, such as low pressure, temperature swings, and 
TABLE II: Available Battery Technologies to Store Energy at CubeSats

\begin{tabular}{|c|c|c|c|c|}
\hline Technology & $\begin{array}{l}\text { Nominal Voltage } \\
\text { (V) }\end{array}$ & $\begin{array}{l}\text { Gravimetric Ca- } \\
\text { pacity }(\mathrm{Wh} / \mathrm{Kg})\end{array}$ & $\begin{array}{l}\text { Recharge Cycle } \\
\text { Life }\end{array}$ & Notes \\
\hline Nickel-Cadmium (NiCd) & $1.25-1.3$ & $40-60$ & 1500 & $\begin{array}{l}\text { Low voltage, very low capacity, high power, memory } \\
\text { effect, and good safety. }\end{array}$ \\
\hline $\begin{array}{l}\text { Nickel Metal Hydride } \\
(\text { MiMH) }\end{array}$ & $1.25-1.3$ & $60-120$ & $300-500$ & $\begin{array}{l}\text { Low voltage, low capacity, good power, memory effect, } \\
\text { and good safety. }\end{array}$ \\
\hline Nickel Hydrogen $\left(\mathrm{NiH}_{2}\right)$ & $1.25-1.3$ & $55-75$ & $>20000$ & $\begin{array}{l}\text { Low voltage, low capacity, high power, and excellent } \\
\text { recharge cycle life. }\end{array}$ \\
\hline $\begin{array}{l}\text { Lithitum Cobalt Dioxide } \\
\text { (LCO) }\end{array}$ & $3.6-3.7$ & $150-200$ & $500-1000$ & $\begin{array}{l}\text { Low charge/discharge rate capability, poor safety, and low } \\
\text { thermal runaway temperature. }\end{array}$ \\
\hline $\begin{array}{l}\text { Lithium Manganese Ox- } \\
\text { ide (LMO) }\end{array}$ & $3.7-3.8$ & $100-150$ & $300-700$ & Low cost, high power, and good safety. \\
\hline $\begin{array}{ll}\text { Lithium } & \text { Nickel } \\
\text { Manganese } & \text { Cobalt } \\
\text { Dioxide (NMC) } & \\
\end{array}$ & $3.6-3.7$ & $150-220$ & $1000-2000$ & $\begin{array}{l}\text { Low charge/discharge rate capability, low resistance, and } \\
\text { good safety. }\end{array}$ \\
\hline $\begin{array}{l}\text { Lithium Iron Phosphate } \\
\text { (LFP) }\end{array}$ & $3.2-3.3$ & $90-120$ & $>2000$ & $\begin{array}{l}\text { Very high discharge capability, low gravimetric capacity, } \\
\text { and very good safety and recharge cycle life. }\end{array}$ \\
\hline $\begin{array}{l}\text { Lithium Nickel Cobalt } \\
\text { Aluminum Oxide (NCA) }\end{array}$ & 3.6 & $200-260$ & 500 & High energy, low cycle life, and low power. \\
\hline
\end{tabular}

outer space radiation, either temporarily or permanently. For example, when the CubeSat is in the eclipse, the temperature may fall down to $-40^{\circ} \mathrm{C}$, which may even cause a temporary battery fail.

Electrical power storage (EPS) performance can be listed as the most important factor that limits mission feasibility and life span of CubeSats. Furthermore, the batteries and the attached power control systems tend to become the heaviest and the biggest part of CubeSats, which increases the cost of deploying CubeSats exponentially. The ever increasing demand of highspeed communication in addition to various different sensors and equipment, such as magnetotorquers, magnetometers, reaction wheels, rate sensors, micro/mini thrusters, and cameras produce an increasing demand of power. In the challenge to fit all these subsystems including the EPS into the smallest possible cube, there is a demand for alternative power systems that would help CubeSats to supply the exponentially increasing demand of very high speed communication.

On the other hand, due to more space in larger LEO satellite systems, more power can be stored by utilizing appropriate rechargeable systems. The power to recharge EPS systems in larger satellites can be generated by large solar arrays or consistent active power generators like a radioisotope generator. With the new advancements in EPS technology, more energy can be stored in a unit mass and unit space. Some advantages can also be provided to mitigate problems against abusive space conditions such as extreme temperatures [8]. For example, hybrid use of Li-ion together with ultracapacitors has been shown to provide some advantages over non-hybrid technologies for high-power pulsed current loads [8]. EPS systems for larger dimensions of larger LEOs can be designed to meet all the required extreme conditions and can store excessive amounts of energy, if designed smartly.

\section{Potential Solutions}

As a potential solution to aforementioned problems, we advocate the wireless power transfer from the bigger LEO satellites, such as from Iridium, to nearby CubeSats. As discussed earlier, bigger satellites have more space to install larger solar panels and hence to produce more power. This power can be transmitted to CubeSats using laser-based technologies such as free-space optics (FSO), which we define as OWPT. In fact, the OWPT is already investigated in the literature to transmit huge amount of power from space to ground.

Indeed, the amount of space-based solar energy is huge. It is estimated that the amount of solar energy in one year is almost equal to the all oil reserves on the earth. Beginning from late 80 s and early $90 \mathrm{~s}$, transmitting the harvested space based solar energy by laser transmission is discussed by many researchers (see [9], [10] and the references therein). Japan Aerospace Exploration Agency (JAXA) has proposed to build up a space-based solar OWPT system delivering 1 GW to an earth station. This justifies the idea of spacebased solar power generation and its transmission to CubeSats using state-of-art OWPT technologies. Another justification of the proposed idea is NASAs practical demonstration of a laser-based communication system between earth and moon at distance of $384,400 \mathrm{~km}$ between them and at a speed of 30 $\mathrm{km} / \mathrm{s}$ and $1.02 \mathrm{~km} / \mathrm{sec}$, respectively.

We propose the concept of power HotSpots around the larger satellites, wherein the OWPT is optimized within the radius $r$. Whenever a CubeSat enters a power HotSpot, it may demand power from the larger satellite either to charge its batteries or to perform different power demanding tasks. Fig. 4 illustrates the concept of power HotSpots in space, where smaller satellites can fulfill their power demands either free of charge or in return of some monetary benefits. The amount of power delivered to CubeSats will depend on many factors, such as power generation capacity of the bigger satellite, the distance between the satellites' orbits $\Delta d$, the direct line of sight distance between them $d_{\text {los }}$, and the power conversion efficiency of transmitting laser and receiving solar panels. We define power HotSpot as a zone, where the power received at a CubeSat is comparable with its demands. The amount of power delivered to CubeSats can be maximized by carefully controlling the following parameters.

The advantages of OWPT in space include, but are not limited to the following.

- The atmosphere between LEOs is so thin that the atmospheric effects, such as scattering, absorption, rain, fog and cloud do not exist.

- The laser beams have a very high directivity compared 


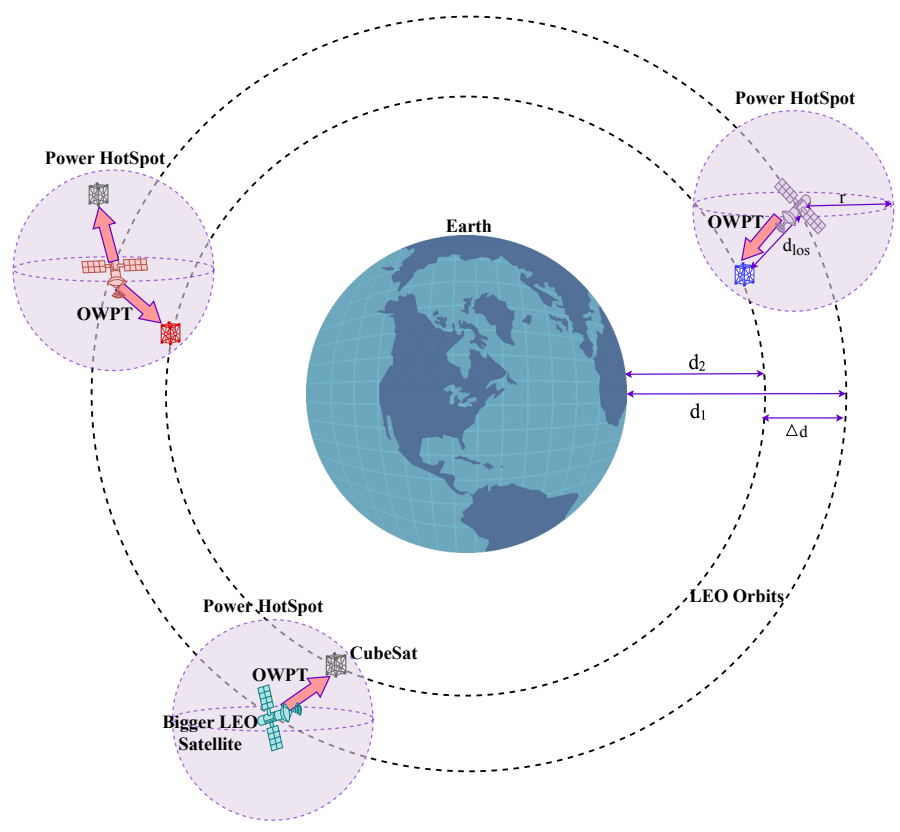

Fig. 4: An illustration of optical wireless power transfer from larger satellites to CubeSats.

to microwave power transmission. Therefore, more power can be efficiently directed to the target receiver.

- CubeSats equipped with solar panels can directly take advantage of the proposed OWPT without the need to change any of its electrical / electronic systems. The solar panels can simply be used to receive the OWPT. If microwave power transmission was used instead, bulky, heavy, complex, and expensive electronic systems would have to be installed.

- Smaller and larger LEOs, which are interconnected with FSO communication links, are already aware of their directions. Therefore, no additional complex algorithms are required for finding directions of the transmitter and the receiver.

- With carefully-designed constellations, there can always be one or more larger LEOs in sight that is ready to provide power to CubeSats.

\section{Optical Wireless POWER Transfer}

In this section, we study the possibility of transmitting excess energy generated by the solar arrays of a larger LEO to a CubeSat by employing OWPT, when required. First, we give an outline of existing state-of-art solar cell technologies and their efficiencies, since they are the most reasonable energy sources of satellites currently. Next, we discuss current and the estimated future conversion efficiencies of OWPT. Finally, we simulate the possibility of a typical OWPT between a larger LEO and a CubeSat.

\section{A. Solar Cells as Energy Sources in Satellites}

We are receiving the solar energy using on-board solar cells for both CubeSats and larger LEO satellites. Most of the cheap Si-based solar cells achieve an overall efficiency between 14\%$19 \%$, which are generally not suitable to space-based radiation.
GaAs-based solar cells are the most preferred solar cells in space since the launch of Venera 3 in 1965, because they are more efficient, less affected by the temperature swing, and degrade more slowly than silicon in the presence of space radiation.

Many new solar cell technologies are currently being developed, such as Perovskite-based and organic solar cells. The best achieved efficiency of single-junction Si-based solar cell is reported to be $25.6 \%$, while it is $24.2 \%$ for a Perovskite-based thin-film solar cell. Currently, the best single-conjunction III-V GaAs-based thin film solar cell has $29.1 \%$ efficiency. One of the best state-of-the-art non-concentrated solar-cell technology based on multi-conjunction structured GaAs-InP cells has been reported to have of $35.8 \%$ efficiency in the space (whereas $38.8 \%$ in terrestrial) [11]. A six conjunction monolithic GaAs based solar cell was recently claimed to have $39.2 \%$ efficiency [12]. This new structure uses AlGaInP / AlGaAs / GaAs / GaInAs materials and achieves an efficiency of up to $47.1 \%$ when concentrator cells are employed [12].

\section{B. Energy Harvesting Efficiency From Larger LEOs to Cube- Sats}

The spectrum of the laser beam originating from larger LEOs to be harvested in CubeSats is completely different than the spectrum of the sun. Lasers produce coherent beams with the same wavelength and the phase. The solar cells at the CubeSat can be optimized to the wavelength of the laser emitted from the larger LEOs for a better conversion efficiency.

It has been discussed in [10] that the highest solar cell efficiency today is $66 \%$ and it will achieve $85 \%$ in the future, when the wavelength of the emitting laser is optimized for the best performance.

It has also been discussed in [10] that the most efficient laser reported has $74 \%$ efficiency and it is expected to achieve 
$85 \%$ in the near future. Furthermore, it has been shown in [10] that for the best overall conversion efficiency, the wavelength of the laser must be chosen in between $500 \mathrm{~nm}$ and $1 \mu \mathrm{m}$. We can conclude that the conversion efficiency of a state-of-art OWPT is currently $49 \%$, however it is estimated to achieve $72 \%$ in the near future [10].

\section{Optical Wireless Power Transfer}

Unlike earth-based atmospheric turbulence and varying weather conditions, such as fog, snow, and haze, among others, these do not exist while employing OWPT in space, thereby allowing a higher visibility range. Hence, the utility of FSO links for energy harvesting purpose becomes much easier allowing for simpler analysis. In addition, OWPT gives an inherent benefit of receiving laser energy on the already installed solar panels on CubeSats. Moreover, based on Table I of [13], we can realize a desired spot diameter of around the length of CubeSats' edge. In such a case, the energy harvesting panels all around the CubeSats will be guaranteed to receive all the transmitted power. In order to obtain such a desired spot diameter for a given range / distance between the LEO satellites and the CubeSats, we can subsequently derive the desired divergence angle as

$$
\Theta=\frac{\text { Spot Size }}{\text { Range }} \text {. }
$$

As a free-space transmission in the LEO orbits, the received free-space optical power, $P_{r}$, can be realized by the following relation [14]

$$
P_{r}=\frac{P_{t} A_{R} \tau_{a} \tau_{T} \tau_{R}}{\frac{\pi}{4} d_{l o s}^{2} \Theta^{2}}
$$

where $P_{t}$ is the transmitted power, $A_{R}$ is the effective receiver aperture area, $\tau_{a}$ is atmosphere transmissivity, $\tau_{T}$ is transmitter transmissivity, $\tau_{R}$ is receiver transmissivity, $d_{l o s}$ is the line of sight distance between the transmitter and receivers satellites, and $\Theta$ is the divergence angle. As a proof of concept, we conduct basic simulations to observe harvested power at CubeSat for its different locations in power HotSpot. The value of $A_{R}$ is taken as 0.01 , while the values of $\tau_{a}, \tau_{T}$ and $\tau_{R}$ are considered as $1,0.85$ and 0.85 , respectively [10]. In addition, by using (2) we can estimate that for a spot size of $10 \mathrm{~cm}$, a divergence angle of $4 \mu \mathrm{rad}$ is needed at the transmitter, if $d_{l o s}$ between the satellites is $25 \mathrm{Km}$.

Fig. 5 presents the relation between transmitted and received signal powers from bigger LEO to CubeSat for different values of line-of-sight distances $\left(d_{l o s}\right)$. We suppose that, $\Delta d$ i.e., the distance between LEO orbits, is $25 \mathrm{Km}$. Hence, the distance between both the satellites cannot go less than this value. The values of $d_{l o s}$ are chosen keeping in view the current and future constellations of LEO satellites given in Table 1 of [15]. It can be observed from the figure that the total received power at a distance of $25 \mathrm{Km}$ is around $0.9 \mathrm{KW}$ when the transmitted power is $1 \mathrm{KW}$, which is enough to charge the batteries given that the OWPT time between the satellites in fair enough. For this, we assume that the satellites are moving in the same direction. At a distance of $25 \mathrm{Km}$, their relative velocity changes with only $11 \mathrm{~m} / \mathrm{s}$. The CubeSat will move 11 $\mathrm{m} / \mathrm{s}$ faster than bigger LEO (see Fig. 4). At this distance, the CubeSat will remain in line-of-sight of the LEO for 17 hours and 18 minutes, approximately. This is sufficient amount of time for a CubeSat to get power from a bigger LEO satellite.

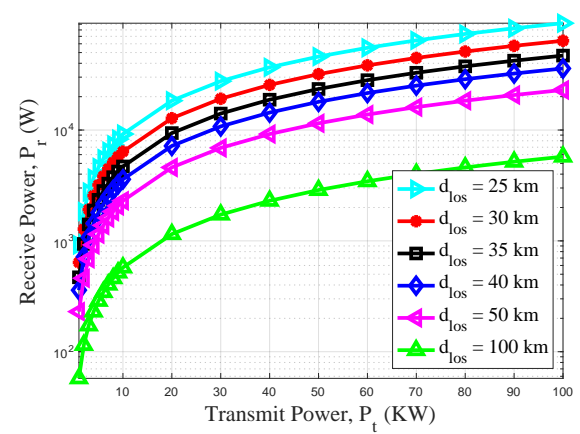

Fig. 5: Received signal power at a CubeSat at different points in power HotSpots.

\section{Future Research Challenges}

1) LoS and Visibility Time: An important parameter in obtaining the maximum energy at CubeSats is the time for which both satellites interact with each other, i.e., the visibility time. This is the time a CubeSat will remain in the power HotSpot. To estimate the visibility time of a CubeSat from a potential LEO, we first need to understand the constellations of different bigger LEO satellites, the constellations of CubeSats, and the launch process of CubeSats. The visibility time between a LEO and a CubeSat mainly depends on the process when the CubeSat is launched, its relative distance from the LEO, and the relative direction of both. For a bigger LEO satellite orbiting at a distance of $725 \mathrm{Km}$ and CubeSat at $700 \mathrm{~km}$ from earth, their visibility time is calculated as 17 hours and 18 minutes (see Section IV-C). From this data, the constellation of bigger LEO and the CubeSat can be planned, such that CubeSat will remain in power HotSpot of satellite.

Given the fact that there are LEO satellites that provide global coverage, there are chances that they will in most cases provide the visibility to many CubeSats lying under their orbit. For instance, Iridium satellites provide global coverage at a height of $781 \mathrm{Km}$. Similarly, Globalstar has 24 LEO satellites orbiting at a distance of $1417 \mathrm{Km}$ from earth at an inclination angle of 52. In order to get a maximum visibility time, the CubeSats may be launched near those orbiting satellites. In addition, some satellites in the LEOs weigh above $3000 \mathrm{Kg}$ with a power generation capacity in kilo Watts. These satellites are a potential source of power to CubeSats.

Furthermore, a careful design of LEO satellites and CubeSats constellation will help to point the laser beam from a larger LEO satellite to a CubeSat. In fact, a motor can be installed on the LEO satellite whose rotation speed can be controlled using the known values of $d_{l o s}$ and the exact speed and trajectory of CubeSat. In particular, the angular speed of the motor will depend on the relative speed of CubeSat with respect to LEO satellite.

To summarize, an open research challenge is to carefully design and plan the constellations of CubeSats and bigger 
LEO satellites, such that the power transfer remains optimized and CubeSats always get power from at least one of the LEO satellites. This will require a sophisticated launch process for CubeSats. However, a careful design of power HotSpot such that the value of $r$ is maximized, and a careful choice of no. of bigger LEOs to cover the global coverage in terms of wireless power transfer, can ease the launch process of CubeSats not requiring them to be in a very precise orbit.

2) Energy from Higher Orbits: This work mainly focuses on OWPT from bigger LEO satellites to CubeSats only, however, this power transmission may also be possible from MEO and GEO satellites, especially when an optical communication link between a GEO satellite and a CubeSat is recently demonstrated [16]. The size of the satellites in higher orbits is generally large and they have more capacity for generating solar power. However, this requires a thorough investigation on the transmission of sufficient amount of power over a distance of 50,000 Kms, which will require a sophisticated equipment not only on CubeSats to efficiently receive this optical power, but also on GEO / MEO satellites to transmit a highly directive and very powerful laser beam.

3) Physical Challenges: There are many physical challenges for an optimized satellite mission. An obvious challenge is towards a more efficient solar cell technology by employing new multi-conjunction solar cell chemistry. Additionally, most of the state-of-art solar cells are optimized to operate on the surface of the earth. However, spectrum of the sunlight in space is different from the spectrum on the surface of the earth, mostly due to the atmospheric effect that absorbs or reflects some of the spectrum acting as an optical filter. Therefore, a challenge is to optimize the solar cell chemistry for the spectrum of the sunlight in the space, to achieve more efficient solar arrays in the satellites.

Furthermore, the employed solar cells in CubeSats must be very efficient when converting the transmitted laser power. Therefore, another challenge is that the employed solar cells must also be optimized with respect to the laser wavelength, or the laser's wavelength must be selected very carefully such that the solar cells in the CubeSat operates most efficiently. It is not feasible to equip CubeSats with two different cell chemistries one optimized for Sun spectrum and the other optimized for the laser beam wavelength. The CubeSats should be equipped with a single type of solar panel optimized for both the Sun spectrum at the space and the laser beam wavelength. One more challenge is to develop more efficient lasers that can convert the electricity to high-power laser beams.

4) End-of-Life of CubeSats and Space Debris: An important challenge is to careful plan the end-of-life of CubeSats so they do not contribute to space debris. A large number of CubeSats and other bigger satellites in LEO orbits increase the chance of collisions in space, hence accelerating the Kessler Syndrome, a chain reaction of orbital collisions which increases the chances of further collision such that LEO orbits become hostile for any spacecraft. Federal Communications Commission (FCC) requires all satellites launched in space to ensure a safe end of their operational life. While GEO satellites generally end in graveyard orbit at the end of their life, the LEO satellites, such as CubeSats would require further study for their end-of-life disposal.

\section{CONCLUSION}

CubeSats consume much more power when they transmit at higher data rates. However, they may fall short of power when communicating at a higher data rate, since they are small and the power generating capability within them is low. There are also bigger LEO satellites in the orbit, which can generate and store much more power.

In this paper, we have introduced the idea of power Hotspots wherein bigger LEOs transfer optical wireless power to CubeSats in need. For this purpose, we have investigated the latest state-of-art technologies that can enable the OWPT in between larger LEOs and CubeSats. Moreover, basic simulations of OWPT between them are presented. We have also highlighted current and future research challenges in the direction of OWPT between larger LEOs and smaller LEOs.

\section{REFERENCES}

[1] E. Yaacoub and M. Alouini, "A key $6 \mathrm{G}$ challenge and opportunityconnecting the base of the pyramid: A survey on rural connectivity," Proceedings of the IEEE, vol. 108, no. 4, pp. 533-582, 2020.

[2] X. Huang, J. A. Zhang, R. P. Liu, Y. J. Guo, and L. Hanzo, "Airplaneaided integrated networking for 6G wireless: Will it work?" IEEE Vehicular Technology Magazine, 2019.

[3] N. Saeed, A. Elzanaty, H. Almorad, H. Dahrouj, T. Y. Al-Naffouri, and M. Alouini, "Cubesat communications: Recent advances and future challenges," To appear in IEEE Communications Surveys Tutorials, 2020.

[4] R. Bassoli, F. Granelli, C. Sacchi, S. Bonafini, and F. Fitzek, "Cubesatbased 5G cloud radio access network: A novel paradigm for on-demand anytime-anywhere connectivity," IEEE Vehicular Technology Magazine, 2020.

[5] T. D. Ponnimbaduge Perera, D. N. K. Jayakody, S. K. Sharma, S. Chatzinotas, and J. Li, "Simultaneous wireless information and power transfer (SWIPT): Recent advances and future challenges," IEEE Communications Surveys Tutorials, vol. 20, no. 1, pp. 264-302, 2018.

[6] P. Bambach, J. Deller, E. Vilenius, S. Pursiainen, M. Takala, H. M. Braun, H. Lentz, and M. Wittig, "DISCUS-The Deep Interior Scanning CubeSat mission to a rubble pile near-Earth asteroid," Advances in Space Research, vol. 62, no. 12, pp. 3357-3368, 2018.

[7] O. Popescu, "Power budgets for CubeSat radios to support ground communications and inter-satellite links," IEEE Access, vol. 5, pp. $12618-12625,2017$.

[8] A. Kuperman and I. Aharon, "Battery-ultracapacitor hybrids for pulsed current loads: A review," Renew. Sust. Ener. Rev., vol. 15, no. 2, pp. 981 - 992, 2011.

[9] L. Summerer and O. Purcell, "Concepts for wireless energy transmission via laser," in International Conference on Space Optical Systems and Applications (ICSOS), 01 2009, pp. 1-10.

[10] T. Miyamoto, "Optical wireless power transmission using VCSELs," in Proc. SPIE, vol. 10682, May 2018.

[11] P. Chiu, D. Law, R. Woo, S. Singer, D. Bhusari, W. Hong, A. Zakaria, J. Boisvert, S. Mesropian, R. King, and N. Karam, "35.8\% space and $38.8 \%$ terrestrial 5J direct bonded cells," in IEEE 40th Photovoltaic Specialist Conference (PVSC), Jun. 2014, pp. 1-3.

[12] J. F. Geisz, M. Steiner, N. Jain, K. L. Schulte, R. M. France, W. McMahon, E. Perl, and D. Friedman, "Building a six-junction inverted metamorphic concentrator solar cell," IEEE J. Photovolt., vol. 8, no. 2, pp. 565-575, Mar. 2018.

[13] A. G. AlKholidi and K. S. Altowij, Free space optical communications - Theory and practices. IntechOpen, Nov. 2014.

[14] F. Kalil, "Optical and microwave communications-A comparison," NASA technical note, 1966.

[15] T. G. Reid, A. M. Neish, T. F. Walter, and P. K. Enge, "Leveraging commercial broadband LEO constellations for navigation," in Proceedings of ION GNSS Conference, 2016, pp. 2300-2314. 
[16] A. Carrasco-Casado, P. Xuan Do, D. R. Kolev, T. Hosonuma, K. Shiratama, H. Kunimori, Y. Abe, S. Nakasuka, and M. Toyoshima, "Intersatellite-link demonstration mission between CubeSOTA (LEO CubeSat) and ETS9-HICALI (GEO satellite)," in 2019 IEEE International Conference on Space Optical Systems and Applications, Portland, Oregon, USA, October 2019, pp. 1-6. 